\title{
Bcl-3 promotes multi-modal tumour cell migration via NF-кB1 mediated regulation of $\mathrm{Cdc42}$
}

\section{Authors:}

Daniel J Turnham ${ }^{1}$, William W Yang ${ }^{2}$, Julia Davies ${ }^{4}$, Athina Varnava ${ }^{1}$, Anne J Ridley ${ }^{3}$, R. Steven Conlan ${ }^{4}$, Richard W.E Clarkson ${ }^{1}$

${ }^{1}$ European Cancer Stem Cell Research Institute, School of Bioscience, Cardiff University, Cardiff, CF24 4HQ, UK

${ }^{2}$ Department of Pathology, UCL Cancer Institute, University College London, London, WC1E 6BT, UK

${ }^{3}$ School of Cellular and Molecular Medicine, University of Bristol, Biomedical Sciences Building, University Walk, Bristol, BS8 1TD, UK

${ }^{4}$ Swansea University Medical School, Singleton Park, Swansea, SA2 8PP, UK

Corresponding author: Richard Clarkson, clarksonr@cf.ac.uk, 02920870249

\begin{abstract}
A key challenge in the implementation of anti-metastatics as cancer therapies is the multimodal nature of cell migration, which allows tumour cells to evade the targeted inhibition of specific cell motility pathways. The NF- $\mathrm{B}$ co-factor B-cell lymphoma 3 (Bcl-3) has been implicated in breast cancer cell migration and metastasis, yet it remains to be determined exactly which cell motility pathways are controlled by Bcl-3 and whether migrating tumour cells are able to evade Bcl-3 intervention. Addressing these questions and the mechanism underpinning Bcl-3's role in this process would help determine its potential as a therapeutic target.
\end{abstract}

Here we identify Bcl-3 as an upstream regulator of the two principal forms of breast cancer cell motility, involving collective and single-cell migration. This was found to be mediated by the master regulator $\mathrm{Cdc} 42$ through binding of the NF- $\mathrm{BB}$ transcription factor $\mathrm{p} 50$ to the Cdc42 promoter. Notably, Bcl-3 depletion inhibited both stable and transitory motility phenotypes in breast cancer cells with no evidence of migratory adaptation. Overexpression of Bcl-3 enhanced migration and increased metastatic tumour burden of breast cancer cells in vivo, while overexpression of a mutant Bcl-3 protein, which is unable to bind p50, suppressed cell migration and metastatic tumour burden suggesting that disruption of $\mathrm{Bcl}-3 / \mathrm{NF}-\kappa \mathrm{B}$ complexes is sufficient to inhibit metastasis. 
These findings identify a novel role for Bcl-3 in intrinsic and adaptive multi-modal cell migration mediated by its direct regulation of the Rho GTPase Cdc42 and identifies the upstream Bcl-3:p50 transcription complex as a potential therapeutic target for metastatic disease.

Keywords- Bcl-3, NF- $\kappa B$, Breast cancer, Metastasis, Migration, Cdc42

\section{Summary}

Overcoming multi-model migration and motility switching represents a key challenge in the implementation of anti-metastatic therapies. Here we characterise the role of Bcl-3 in this process and identify its interaction with p50 as a potential therapeutic target for metastasis.

\section{Introduction}

The process of cell migration occurs in a number of normal physiological and pathological contexts with cells capable of utilising a variety of mechanisms to promote their movement either as single cells or as a collective entity [2-4]. Cell migration is primarily driven through Rho GTPase-regulated signalling networks which mediate dynamic changes in the cytoskeleton and cell adhesions to produce movement. Cdc42 has been identified as a key mediator of this process primarily through regulating the extension of lamellipodia and filopodia in both single cell and collective migration $[2,5,6]$. Alternatively single cells can also be driven by high levels of Rho/ROCK signalling to propel more rounded cells through actomyosin contractions [7], with Cdc42-MRCK signalling also known to regulate this propulsion independently of ROCK to drive cell invasion $[8,9]$.

Invading cancer cells are able to utilise these different mechanisms and in metastatic breast cancer both collective and single cell motility has been observed [3, 10-13]. Although metastasis is a complex multi-step process it fundamentally relies on the movement of tumour cells from primary to secondary sites, with cells often travelling relatively large distances around the body. There are currently no curative treatments for metastatic breast 
cancer making it responsible for almost all breast cancer-associated deaths, which is one of the most common causes of death from cancer in the UK [14]. Current standard of care for metastatic breast cancer focuses on improving quality of life, yet with over $90 \%$ of all deaths from solid cancers attributed to metastasis it has been argued that existing therapies aimed at regressing primary tumours should be complemented with anti-metastatic drugs to prevent the initial or ongoing dissemination of disease as early as possible $[15,16]$. Candidate antimetastatic interventions include the recently coined 'migrastatics', which target the fundamental mechanisms that drive cell migration [15]. However, to ensure successful translation into a clinical setting, such interventions must overcome the dual problems of nonselective toxicity and cellular plasticity, whereby cells within the tumour are able to evade inhibition of specific migration pathway inhibitors by adopting alternative modes of migration [15]. Indeed the ability of cancer cells to switch between different types of motility depending on their environment has made migratory cellular plasticity one of the main concerns regarding anti-metastatic intervention, as it may lead to treatment resistance and phenotypic evasion if all migratory mechanisms are not simultaneously inhibited $[15,17]$. Despite some promising advances in this area, including next generation ROCK inhibitors [18] and agents targeting the actin cytoskeleton [19-21], ongoing issues of toxicity and efficacy warrant further studies to identify novel targets of multi-modal cell migration.

A new anti-metastatic target which has shown early promise in the pre-clinical setting is the proto-oncogene B-cell lymphoma $3(\mathrm{Bcl}-3)[22,23]$. Bcl-3 is an atypical member of the IKB family of NF- $\mathrm{kB}$ regulators capable of regulating transcription through its interactions with p50 and p52 homodimers as well as acting independently of NF- $\mathrm{B}$ to mediate a number of processes including proliferation, apoptosis and metastasis [22, 24, 25]. Bcl-3 has more recently been implicated in the progression and resistance to therapy in a number of solid tumour types including colorectal, nasopharyngeal, prostate and glioblastoma [26-29]. In 
breast cancer, high expression of Bcl-3 has been attributed to a reduction in metastasis-free survival [22], while Bcl-3 inhibition has been shown to reduce metastatic tumour burden in both triple negative and ERBB2-driven tumour models in vivo [22, 23]. Importantly, complete deletion of Bcl-3 in these mouse models resulted in viable animals with only a minor disruption to the humoral immunological compartment [30] suggesting that the targeted inhibition of Bcl-3 in humans may be well tolerated. These anti-metastatic effects following Bcl-3 suppression have been attributed to a reduction in cell migration associated with the downregulation of TGF- $\beta$ signalling [23], yet its potential to inhibit the tumour cell's capacity to stably or transiently adopt one or more modes of cell migration, which represents a major clinical hurdle, has not been investigated $[5,31]$.

In this study we use three cancer cell lines with distinct migratory properties to show how suppression of Bcl-3 using siRNA can reduce three different forms of cell motility, primarily

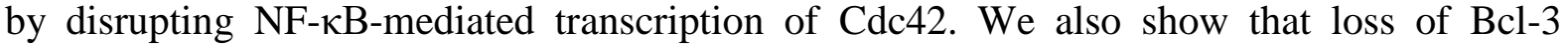
maintains its inhibitory effects in cells actively switching motility modes during EMT, while overexpression of Bcl-3 promotes migration and metastatic potential in vivo. Together this study highlights the therapeutic potential of Bcl-3 as an anti-metastatic target.

\section{Materials and methods}

\section{Cell lines and reagents}

Verified human breast cancer cell lines MDA-MB-231 ${ }^{\text {ER-HER2- }}$, MDA-MB-436 ER-HER2- and MCF-7 ${ }^{\text {ER+HER2- }}$ cells were obtained from ATCC and used within 6 months and a maximum of 10 passages after resuscitation. Each cell line was authenticated by short tandem repeat profiling as described by the ATCC cell bank and regularly tested for mycoplasma contamination. MDA-MB-231-luc-D3H2LN cells were derived from a spontaneous lymph node metastasis from an originally verified MDA-MB-231-D3H1 mammary fat pad 
tumour by Caliper Life Sciences (Perkin Elmer Inc.). All cell lines were maintained in RPMI 1640 GlutaMAX media (Invitrogen) supplemented with $10 \%$ foetal bovine serum (Invitrogen) at $37^{\circ} \mathrm{C}$ in $5 \% \mathrm{CO}_{2}$.

Cell lines were transfected with Bcl-3 or Cdc42 siRNA using a mixture of 4 small interfering RNAs (siRNA) each designed to suppress their respective targets. Bcl-3 siRNA 1: 5'AGACACGCCUCUCCAUAUU-3', , siRNA 2: 5'-GGCCGGAGGCGCUUUACUA-3', SiRNA $\quad 3: \quad$ 5'-GCGCAAAUGUACUCCGGCA-3', $\quad$ siRNA $\quad 4: \quad$ 5'GCCGGGAGCUCGACAUCUA-3'. Cde42 siRNA 1: 5'-CGGAAUAUGUACCGACUGU3', $\quad$ siRNA $2: \quad$ 5'-GCAGUCACAGUUAUGAUUG-3', $\quad$ siRNA $\quad 3: \quad$ 5'GAUGACCCCUCUACUAUUG-3', siRNA 4: 5'-CUGCAGGGCAAGAGGAUUA-3' (ONTarget plus SMART pool, Dharmacon). A pool of control RNAs (scRNA) designed to have minimal effect on human gene expression was used to control for transfection (scRNA 1- 5'UGGUUUACAUGUCGACUAA-3', scRNA 2- 5'- UGGUUUACAUGUUGUGUGA-3', scRNA 3- 5'- UGGUUUACAUGUUUUCUGA-3', $\quad$ scRNA $\quad 4-\quad 5$ 'UGGUUUACAUGUUUUCCUA-3') (Dharmacon). Wildtype Bcl-3 and ANK mutant Bcl-3 plasmids were a kind gift from Dr. Alain Chariot (University of Liège, Belgium). Transfection was performed on $70 \%$ confluent adherent cells using lipofectamine 3000 (Invitrogen) and serum-free Opti-MEM (Invitrogen) according to manufacturer's instructions.

\section{Bioinformatics analysis}

Gene expression data of 56 breast cancer cell lines was obtained from the cell line encyclopedia using cBioPortal [32, 33] and was used for quantifying EMT using the previously reported EMT scoring tool [34]. Samples with a significant p-value $(<0.05)$ and a positive score were determined as mesenchymal, whereas samples with a significant p-value 
and a negative score were determined as epithelial. Samples with a p-value that did not reach a significant level were considered as intermediate.

Analysis of affymetrix experiments were carried out in the GeneSpring GX Pathway Architect software. The ExonRMA summarisation algorithm was performed on all projects with the baseline set to the median of all samples. The samples were quality checked by assessing PCA values to ensure appropriate clustering. Probe sets were filtered based on their signal intensity values and 43590 out of 48144 entities had values between 20 and 100 percentiles. The Benjamini and Hochberg False Discovery Rate multiple testing correction was performed to determine corrected p-values and entities were filtered based on a cut-off of p-value $<0.05$.

\section{EMT stimulation}

EMT stimulation was performed using StemXVivo media supplement (R\&D systems) which contains recombinant human Wnt-5a protein, recombinant human TGF-betal protein, antihuman E-cadherin antibody, anti-human sFRP-1 antibody and anti-human Dkk-1 antibody. Cells were treated with StemXVivo for $48 \mathrm{~h}$ days before media was replaced with fresh EMT inducing supplement for a further 3 days.

\section{Cell viability assay}

To determine cell viability Cell Titre Blue (CTB) assay (Promega) was used on cells grown in 96-well plates. On the day of analysis, $20 \mu \mathrm{L}$ of CTB reagent (Promega) was added to 100 $\mu \mathrm{L}$ of growth medium in each well. After $1 \mathrm{~h}$ of incubation at $37^{\circ} \mathrm{C}$ in $5 \% \mathrm{CO}_{2}$ fluorescence intensity was measured at 560/590 nm using a ClarioStar plate reader (BMG Labtech).

\section{Transwell migration assay}

To assess chemotaxis driven migration Fluoroblok migration assays (Corning) were used. $5 \mathrm{x}$ $10^{3}$ cells diluted in serum free growth medium were added to the top chambers of a 96-well 
Fluoroblok culture plate (Corning) before $200 \mu \mathrm{L}$ of normal growth medium containing FBS was added to the bottom chambers. After $24 \mathrm{~h}$ of incubation the bottom wells were replaced with $1 \mu \mathrm{M}$ Calcein AM (eBioscience) and incubated for $1 \mathrm{~h}$ at $37^{\circ} \mathrm{C}$. Fluorescence intensity of each well was then read at $500 \mathrm{~nm}$ from the bottom of the plate using a Clariostar plate reader (BMG Labtech).

\section{Random single cell migration assay}

To assess random single cell migration, real-time single cell migration assays were performed. Cells were plated at a density of $5 \times 10^{4}$ cells/well into 12 -well plates and left to adhere overnight. Cells were then imaged over an $18 \mathrm{~h}$ period using a time-lapse incubation chamber (Leica) and analysed using CellTracker image processing software [35] semiautomatically with a minimum of 50 cells per condition analysed. Dividing cells were removed from the data set, and any tracks not-matching cell movement manually adjusted.

\section{Wound healing assay}

To assess collective migration transwell assays were performed. Confluent cell monolayers were scratched using $10 \mu \mathrm{L}$ pipette tips before cells were washed gently with PBS to remove floating cells. Pictures were then taken on an inverted microscope (Leica) at 4 different fields of view at $0 \mathrm{~h}$ and $24 \mathrm{~h}$ after initial scratches were made. Images were analysed using ImageJ to measure changes in the total area covered by cells.

\section{G-LISA assay}

To determine changes in Rho-GTPase activity G-LISA GTPase activation assays (Cytoskeleton) were performed. Cell lysates were harvested on ice by cell scraping with 250 $\mu \mathrm{L}$ of lysis buffer including protease inhibitor (Cytoskeleton) before being snap frozen in liquid nitrogen. Protein quantification, antigen retrieval, antibody incubations and detection were all performed following manufacturer's instructions. 


\section{Immunofluorescence}

Cells were grown on glass coverslips inside of 6 -well culture plates and fixed in $4 \%$ formalin for 15 min before being washed $3 \mathrm{x}$ in PBS. Cells were permeabilized using $0.2 \%$ triton-X100 (Sigma) followed by $30 \mathrm{~min}$ blocking in 1\% BSA (Sigma) in PBS. Cells were then incubated in primary antibody overnight at $4^{\circ} \mathrm{C}$ for either Bcl-3-1:100 (C-14, Santa Cruz), Vimentin-1:200 (V9, Santa Cruz) or E-cadherin 1:200 (C-36, BD biosciences). Following 3x PBS washes fluorescence-conjugated secondary antibodies (Abcam) diluted 1:400 were added alongside Phalloidin-Atto 565 (Sigma) (1:400) and incubated for $1 \mathrm{~h}$ at room temperature. Cells were then stained with DAPI (Invitrogen) for $15 \mathrm{~min}$ and washed $3 \mathrm{x}$ in PBS before being mounted in Mowiol solution (Sigma). Visualisation of cells was performed on a Leica confocal microscope.

\section{qRT-PCR analysis}

RNA extraction was performed using the Qiagen RNEasy kit following the manufacturer's instructions. The concentration and quality of RNA was analysed using a nanodrop 3000 spectrophotometer (ThermoScientific) before being used to synthesise cDNA using the QuantiTect Reverse Transcription kit (Qiagen) following manufacturer's instructions. TaqMan probes were purchased from ThermoFischer Scientific: CDH1-Hs01023894_m1, CDH2-Hs00983056_ml， VIM- Hs00958111_m1， TWIST1- Hs01675818_s1， AxlHs01064444_m1, ZEB1- Hs00232783_m1, Bcl-3- Hs00180403_ml, ACTBHs99999903_m1. Multiplex PCR reactions were performed using TaqMan Universal Master

mix (ThermoFischer Scientific) and run on a QuantStudio 7 Real-Time PCR machine (Applied Biosystems). 


\section{Chromatin immunoprecipitation (ChIP)}

ChIP assay was performed using the Chromatrap kit (Chromatrap) with anti-p50 antibody (Cell Signalling). Cells were cross-linked with formaldehyde and sonicated to an average size of 300-to-500 bp. Lysates were added to microwells immobilized with corresponding antibody. Cross-linked DNA released from the protein-DNA complex was purified and the eluted DNA was further detected by qRT-PCR. Input and IgG was used simultaneously to confirm that the detected signals were derived from the specifically bonding of chromatin and p50 protein.

\section{NF-кB luciferase reporter assay}

The NF- $\mathrm{BB}$ activity of cells was quantified using a luciferase reporter plasmid. Cells were transfected with 900ng of 3x kB luciferase reporter plasmid and 100ng of pcDNA 3.1-LacZ plasmid using lipofectamine 3000. After 48 hours post-transfection cell were lysed using Glo-lysis buffer (Promega) and transferred to a white walled 96-well plate containing BetaGlo or Bright-Glo (Promega) to detect LacZ or luciferase activity respectively. Luminescence relative to LacZ was read using a Flurostar Optima plate reader (BMG Labtech) and displayed as relative light units (R.L.U).

\section{Western Blot}

Total cellular proteins were extracted from cultured cells and analysed by Western blotting using antibodies specific for Bcl-3 (Proteintech, 23959-1-AP) and GAPDH (Santa Cruz Biotechnology; sc365062) and horseradish peroxidise- conjugated secondary antibody (Dako). Antibody binding was detected using ECL prime detection reagent (Amersham) before being developed in a Biorad Chemidoc MP Imaging System. 


\section{Animal metastasis studies}

All mouse experiments were performed in accordance with the Animals (Scientific Procedures) Act 1986 guidelines and approved by the UK Home Office under project licence PPL 30/2849. Animal and cell work included in this project was approved by the Cardiff University Genetic Modification Scientific Committee. Experimental metastasis was established by tail vein injection of $2 \times 10^{5}$ MDA-MD-231-Luc cells. Cells were suspended in $100 \mu \mathrm{L}$ of RPMI media and injected via the tail vein of 8-week old female Hsd: Athymic Nude-Foxn $1^{\text {nu }}$ mice (Harlan Laboratories). Mice were sacrificed 6-weeks after tumour cell injection and organs were immediately harvested and fixed in $4 \%$ formalin. Tissues were then sectioned and subject to H\&E staining before being scanned on Axioscan Z1 (Zeiss) and analysed for metastasis. Cdc42 staining was performed using anti-Cdc42 primary antibody (HPA069590, Sigma) 1:200 following heat-induced epitope retrieval on 231-Luc orthotopic tumour sections.

\section{Statistical analysis}

Error bars on all graphs represent standard error values with the exception of gene expression data which are represented by $95 \%$ confidence intervals. For all tests statistical analysis was performed on raw data obtained from a minimum of 3 independent experiments. Experimental data was analysed in R studio [36] using a repeated measure two-way ANOVA (RM-ANOVA) or an unpaired students $t$ test to determine statistical differences. For gene expression analysis significance was determined using the $95 \%$ confidence interval overlap rule described in [1]. 


\section{Results}

\section{Bcl-3 suppression inhibits three forms of cell migration}

Invasive tumour cells possess the ability to switch between different migratory phenotypes in response to changing microenvironments and pharmaceutical intervention, making this early step in the metastatic process difficult to target and inhibit [17, 37]. Many studies of presumptive cell motility inhibitors often only report the anti-migratory effects of one form of cell migration which may result in resistance when tested clinically. To more robustly address the effects of Bcl-3 inhibition on migration, we assessed the migratory phenotypes of three breast cancer cell lines which, based on their morphological phenotype and previous reports of motility-characteristics $[38,39]$, we hypothesised to migrate differently.

As cells can migrate using either individual or collective cell movement, cell lines were selected based on distinct morphological features. The mesenchymal-like cell line MDA-MB436, which lacks stable cell-cell contacts through E-cadherin [40], was selected as a model for single cell migration; the epithelial-like MCF-7 cell line, which has stable E-cadherinmediated cell-cell contacts [40], was chosen as a model for collective migration; and the MDA-MB-231 cell line was used as an intermediate cell line due to its reduced spindle-like appearance compared to MDA-MB-436 cells but lack of stable cell-cell contacts [40, 41]

(Fig 1A). These cell characteristics were confirmed through immunofluorescence detection of E-cadherin and Vimentin to distinguish epithelial and mesenchymal phenotypes (Supplementary Fig 1A).

To correlate these molecular and morphological characteristics with a migratory phenotype each cell line was compared using three different migration assays designed to evaluate different modes of motility (Fig 1B-D). Single cell chemotaxis-driven motility was tested using Transwell assays in which migrating cells pass through $8 \mu \mathrm{m}$ pores following 
chemotactic stimulation. MDA-MB-231 cells migrated significantly better than both MDAMB-436 and MCF-7 cells, with the latter barely capable of migrating through the chamber pores (Fig 1B). Random single cell migration was then assessed using time-lapse microscopy with MDA-MB-436 cells migrating faster and further than MDA-MB-231 cells, with epithelial MCF-7 cells unable to migrate individually (Fig 1C \& Supplementary Fig 1B). Finally, wound healing assays, which have previously been used to track collective cell motility in breast cancer cell lines [42-46], were used to model the $2 \mathrm{D}$ directional movement of cohesive cells. MDA-MB-436 cells showed a significantly reduced rate of cohesive motility compared to MDA-MB-231 and MCF-7 cells which showed similar rates of collective motility, confirming previous observations that these cells are better suited to single-cell movement (Fig 1D \& Supplementary Fig 1C).

To determine whether Bcl-3 expression associated with a specific morphological subtype and/or mode of motility, relative Bcl-3 mRNA expression was initially compared across 56 breast cancer cell lines from the cancer cell line encyclopedia [33] grouped into epithelial, intermediate or mesenchymal phenotypes based on their baseline genetic EMT score [34]. Ecadherin expression was significantly downregulated in mesenchymal cell lines as expected, but no significant association was observed between Bcl-3 and cell phenotype, indicating that Bcl-3 is ubiquitously expressed across a range of cell types (Fig 1E\&F). Expression of Bcl-3 was confirmed at the protein level in the three cell lines described above with nuclear (functionally relevant) Bcl-3 detected in more than $95 \%$ of the cells within each of the cell lines (Fig 1A). A modest difference in nuclear staining observed between these cell lines was confirmed at the mRNA level, where a small but significant difference in Bcl-3 expression correlated with their ability to undergo random single-cell migration (Fig 1G). No correlation was observed in either chemotaxis-driven or collective migration (Supplementary Fig $2 \mathrm{~A} \& \mathrm{~B})$. 
We wished to investigate the significance of these findings to determine whether the weak correlation with the mesenchymal-like MDA-MB-436 cell line signified a preferential role for Bcl-3 in this form of cell migration or whether the ubiquitous expression of Bcl-3 in all cell types indicated a more universal role for Bcl-3 in cell-specific migratory phenotypes. Bcl-3 was suppressed in each cell line using a pool of 4 Bcl-3 targeting siRNAs which resulted in a significant reduction of Bcl-3 expression compared to scRNA treated controls (Supplementary Fig 3A), with no concomitant change in cell viability observed in any of the cell lines (Supplementary Fig 3B-D). Loss of Bcl-3 expression resulted in a significant reduction in chemotaxis-driven single cell motility in all cell lines (Fig 2A-C), with random single cell motility reduced in both MDA-MB-231 and MDA-MB-436 cells (Fig 2D\&E). No change was observed in MCF-7 cells as they lack the ability to migrate individually in this assay and remained static (Supplementary Fig 3E). Bcl-3 depletion also resulted in a significant reduction in $2 \mathrm{D}$ collective migration in each cell line compared to scRNA treated control cells (Fig 2F-H). The Bcl-3 specific effect of the siRNA pool used in each assay was confirmed by determining the effects of each individual siRNA on MCF-7 cells. Only 3/4 individual siRNAs were capable of significantly suppressing Bcl-3 expression with a significant reduction in migration only observed when Bcl-3 was depleted (Supplementary Fig $3 F \& G)$. Together this data highlights at least three different forms of cell migration that are inhibited through Bcl-3 suppression with no compensatory increases in alternative cell motility.

\section{Bcl-3 is involved in regulating migration during EMT}

Having established that Bcl-3 regulates cell motility in three different assays, we next sought to determine whether these effects were maintained in tumour cells undergoing a phenotypic switch which altered their mode of motility. 
Epithelial to mesenchymal transition (EMT) was initiated in the epithelial-like MCF-7 cell line through a TGF- $\beta$ based conditioned cell culture medium (M-EMT) which also included recombinant-Wnt-5a and anti-E-cadherin antibodies as previously described [47, 48]. Following stimulation with M-EMT for 6 days a large proportion of MCF-7 cells acquired a mesenchymal-like phenotype consisting of spindle-like protrusions and a loss of membrane bound E-cadherin similar to the phenotype observed in mesenchymal MDA-MB-231 cells (Fig 3A). When M-EMT was removed these cells returned to a normal epithelial-like morphology within 4 days indicating a reversion back to an epithelial-like state through mesenchymal to epithelial transition (MET) (Fig 3A). Although Bcl-3 had previously been shown not to correlate with stable epithelial or mesenchymal phenotypes (Fig 1F) we hypothesised that TGF- $\beta$ driven M-EMT stimulation would lead to an increase in Bcl-3 expression due to the known function of Bcl-3 in regulating TGF- $\beta$ driven transcription [22]. As expected, Bcl-3 expression increased following M-EMT treatment (Fig. 3B) coinciding with an increase in EMT markers N-cadherin, Twist, Vimentin, ZEB1 and Axl and loss of membrane bound E-cadherin (Supplementary Fig 4A), returning to unstimulated levels after removal of M-EMT. Overexpression of Bcl-3 or suppression by siRNA under these conditions impacted on only a proportion of these EMT markers suggesting that Bcl-3 was not a master regulator of epithelial to mesenchymal transition but may instead impact on one or more of the transcriptional regulators directly (Supplementary Fig 4B\&C).

The mobility of M-EMT maintained MCF-7 cells was compared to unstimulated MCF-7 cells in each migratory assay described above. M-EMT maintained cells exhibited small increases in single cell migration and a concomitant reduction in collective migratory ability compared to unstimulated cells (Supplementary Fig 4D-F). Time-lapse observations in wound healing assays identified a significant proportion of single highly-migratory cells escaping from the collective cell sheet after M-EMT induction (Fig 3C\&D). When Bcl-3 was inhibited, the 
number of these highly-migratory 'escapees' was significantly reduced (Fig 3E), as was the overall ability of these transitioned MCF-7 cells to migrate in a chemotaxis driven manner (Fig 3F), suggesting that the induction of single cell migration following EMT of epitheliallike cancer cells could also be inhibited by Bcl-3 suppression. The suppression of collective migration by Bcl-3 siRNA was also preserved in M-EMT induced cells (Fig 3G).

\section{Bcl-3 regulates Cdc42 to drive multi-modal cell migration}

We next sought to identify the underlying processes by which $\mathrm{Bcl}-3$ contributed to these different forms of cell migration. To do this we initially performed a global gene expression screen of MDA-MB-231- luc-D3H2LN (231L) cells treated with Bcl-3 siRNA. This cell line was chosen because it exhibited elevated Bcl-3 expression and single-cell migration properties compared to its MDA-MB-231 parental control (Supplementary Fig 5A-C). Subsequent ontological analyses of the microarray data identified Rho GTPase signalling as one of the most significantly affected pathways (Table 1). Of the 27 genes from this pathway differentially regulated following Bcl-3 suppression, the key GTPase family member Cdc42 exhibited the largest change. Downregulation of Cdc42 expression following Bcl-3 suppression was subsequently confirmed in 231L, MDA-MB-436, MCF-7 and M-EMT induced MCF-7 cells (Fig 4A).

As Bcl-3 is a known NF- $\mathrm{BB}$ co-factor we next sought to determine whether loss of Bcl-3 was capable of altering Cdc42 transcription through a specific p50/p65 regulator element identified in the 5'UTR of the Cdc42 gene (Fig 4B). ChIP analysis demonstrated an almost complete loss of p50 binding at the Cdc42 promoter in Bcl-3 suppressed 231L cells (Fig 4C) suggesting that $\mathrm{Bcl}-3$ transcriptional regulation of the native $\mathrm{Cdc} 42$ locus was mediated via

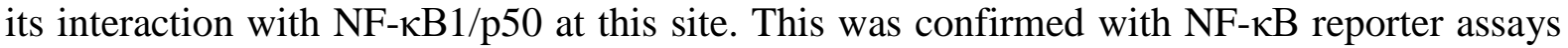
demonstrating a significant reduction in NF- $\mathrm{BB}$ activity in both MDA-MB-436 and 231L 
cells following Bcl-3 inhibition (Fig 4D). No change in NF- $\mathrm{BB}$ activity was observed in MCF-7 cells (Supplementary Fig 5D) which is likely due to the well documented low basal expression of NF-אB in ER-positive breast cancers including MCF-7 cells making it difficult to ascertain a significant difference following Bcl-3 knockdown [49-51]. We next investigated whether the decrease in $\mathrm{Cdc} 42$ expression in Bcl-3-inhibited cells impacted on the levels of functional GTP-bound Cdc42. The proportion of activated GTP-Cdc42 in Bcl-3 suppressed cells was significantly reduced in MDA-MB-436 and showed a similar trend in 231L and MCF-7 cells (Fig 4E) when compared to scRNA treated control cells. Final confirmation of Bcl-3 specific suppression of $\mathrm{Cdc} 42$ was determined by quantitative PCR of its downstream target Cofilin 2 which is negatively regulated by $\mathrm{Cdc} 42$ and was significantly upregulated in our microarray analysis (Table 1). Knockdown of Bcl-3 resulted in a significant upregulation of Cofilin 2 in each of the previously tested cell lines (Fig 4F).

To determine whether loss of Cdc42 expression could account for the observed effects of targeting Bcl-3, without compensatory increases in alternative cell motility, different modes of migration were tested in each cell line. Following suppression of Cdc42 using pooled siRNA (Supplementary Fig 5E) chemotaxis driven and random single cell migration was reduced in MDA-MB-231 and MDA-MB-436 cells respectively (Fig 4G\&H). A significant reduction in 2D collective migration was also observed in MCF-7 cells (Fig 4I). Each cell line was also tested for changes in alternative forms of migration, with similar reductions to Bcl-3 depletion and no compensatory increases observed (Supplementary Fig 5F-K). While attempts to rescue Bcl-3 mediated suppression of migration through overexpression of Cdc42 was compromised by the detrimental effects of ectopic Cdc42 expression in both control and Bcl-3 depleted cells (data not shown), the combined suppression of Cdc42 and Bcl-3 in the same cell line demonstrated that these genes share a common outcome (Fig 4H\&I). 


\section{Reducing functional Bcl-3 decreases cell migration and metastatic spread in vivo}

Having established Bcl-3 as a regulator of multi-modal cell migration we next sought to confirm Bcl-3 as a suitable anti-metastatic target using in vivo models of metastasis.

Initially, to confirm the direct role of Bcl-3 in metastasis, wildtype Bcl-3 (WT Bcl-3) was overexpressed in both 231L and MDA-MB-436 cells and its relative effect on cell migration in vitro and metastatic tumour burden in vivo compared to cells expressing endogenous levels of Bcl-3 was determined (Fig 5) (Supplementary Fig 6). Overexpression of Bcl-3 resulted in an increase in NF- $\kappa \mathrm{B}$ activity (Fig 5A\&B) and single-cell motility in vitro with a concomitant reduction in collective migration (Fig 5C-E). Metastatic tumour burden was also significantly increased in cells overexpressing Bcl-3, manifesting in increased tumour density in lungs and liver compared to controls, and a broader dissemination of tumour foci to include bone and heart (Fig 5F\&G). Although the basal expression of Cdc42 expression remained unchanged (Supplementary Fig 6B), elevated expression of Cdc42 was observed in a subpopulation of tumour cells within Bcl-3 overexpressing tumours compared to parental 231L controls suggesting a transient increase in migrating cells (Supplementary Fig 6C).

In order to determine whether functional targeting of Bcl-3:NF- $\kappa \mathrm{B}$ complexes could be a viable therapeutic option, a protein binding mutant of Bcl-3 (ANK Bcl-3) was overexpressed in the breast cancer cell lines. This mutant form of Bcl-3 has been shown to be unable to bind p50 and p52 homodimers $[52,53]$ and acts as a dominant negative mutant for endogenous Bcl-3 activity [54]. Overexpression of ANK Bcl-3 resulted in a significant reduction in NF$\kappa \mathrm{B}$ signalling, $\mathrm{Cdc} 42$ expression and all three modes of cell migration in vitro (Fig 5B-E) (Supplementary Fig 6B). Accordingly, the metastatic potential of ANK Bcl-3 231L cells was significantly diminished (Fig 5F) with both the density and tissue tropism reduced compared to the parental cell line with endogenous Bcl-3 expression. Furthermore, Cdc42 expression 
was diminished in tumours overexpressing the Bcl-3-ANK mutant compared to controls (Supplementary Fig 6C). The effect of Bcl-3 suppression on experimental metastasis was confirmed in mice injected with $231 \mathrm{~L}$ cells pre-treated with siRNA targeting Bcl-3, indicating that transient suppression of endogenous Bcl-3 levels was also sufficient to reduce metastatic burden in this model of triple-negative breast cancer (Fig 5G\&H).

\section{Discussion}

The identification of novel therapies to target the metastatic process offers the opportunity to improve long-term survival for breast cancer patients either with high risk of developing metastatic disease or for those in which the disease has already progressed $[55,56]$. One of the biggest challenges in targeting potential anti-metastatic candidates is overcoming the diverse and interchangeable migratory potential of metastatic cells, which can be used to overcome various micro-environmental obstacles [17]. Here we show that suppression of Bcl-3 can inhibit at least two distinct modes of cell motility, irrespective of whether this was inherent to the tumour cell subtype or transiently induced by the tumour cell microenvironment. This pan-migratory effect was determined to be a result of downregulated Cdc42 expression and subsequent loss of activity through disrupted NF- $\mathrm{kB}$ signalling on the Cdc42 promoter. Cdc42 is a key regulator of actin cytoskeletal organization which drives cell motility in both individual and collectively migrating cells, making this essential mechanism an important therapeutic target for potential migrastatic candidates $[6,8,15,57,58]$. Although constitutive upregulation of $\mathrm{Cdc} 42$ by Bcl-3 was not observed, likely due to an intolerance of prolonged Cdc42 expression, a subset of tumour cells overexpressing Bcl-3 were shown to express high levels of $\mathrm{Cdc} 42$ suggesting a transient increase in migrating cells and supporting the established role of $\mathrm{Cdc} 42$ in the dynamic processes controlling cell migration [59, 60]. Moreover, Bcl-3 suppression resulted in the significant alteration of 26 other Rho GTPase signalling network genes which although not investigated here are likely 
to also contribute to Bcl-3 driven migration. This builds upon previous data showing the guanine nucleotide exchange inhibitor (GDI) Arhgdib, which preferentially binds to both Rac1 and Cdc42 to regulate cell migration, is upregulated after Bcl-3 inhibition in murine models of breast cancer [23].

Although this study highlights the potential therapeutic benefits of targeting Bcl-3, there are currently no approved Bcl-3 specific targeted therapies, and current technologies limit the potential use of siRNA-mediated Bcl-3 inhibition in a clinical setting. Given that the antimigratory effects observed were dependent on a disruption to the Bcl-3:p50 interaction which manifested in a reduction in metastatic tumour burden in vivo (Fig 4C \& 5F), the development of small-molecule inhibitors capable of blocking this interaction may offer therapeutic benefits in the future. Development of such molecules will require continued characterisation of Bcl-3-mediated migration utilising new 3D platforms capable of closely mimicking the tumour microenvironment which may alter the migratory potential of disseminating tumour cells $[15,61]$. Furthermore, in order to translate this into a clinical setting the most effective and suitable time point for targeting Bcl-3 must also be identified, with suggestions that suitable migrastatics should be administered long-term as early as possible alongside existing anti-cancer therapies which also target the bulk tumour mass [15]. Previous demonstration of the lack of systemic toxicity in Bcl-3-null mice supports the potential efficacy of this approach [30].

The early administration of migrastastatic compounds is also likely to reduce the early acquisition of pro-invasive properties during transitional EMT. Here we found Bcl-3 to be upregulated during this process, which is likely due to its role in regulating downstream TGF$\beta$ signalling targets [22]. Suppression of Bcl-3 during EMT was found to inhibit the upregulation of N-cadherin, Vimentin, Twist and Axl thus partially disrupting EMTassociated transcription while overexpression of Bcl-3 alone was able to directly induce both 
Vimentin and Axl expression. Whether these effects were a result of disrupted TGF- $\beta$ or NF$\kappa \mathrm{B}$-mediated transcription was not investigated here however, $\mathrm{N}$-cadherin has been shown to be directly regulated through Bcl-3 in melanoma cell lines [62] while Vimentin and Twist are known targets of SMAD3-mediated transcription therefore both models are possible [63, 64].

In conclusion the data presented here provide evidence for a non-redundant role for Bcl-3 in mediating multiple modes of breast cancer cell migration through the regulation of $\mathrm{Cdc} 42$. We also confirm Bcl-3 as a driver of metastasis supporting its candidacy as an anti-metastatic target and identify its interaction with p50 as a future therapeutic drug target to mimic the effects of Bcl-3 inhibition. Additional studies are now required to identify the active sites on the Bcl-3 protein responsible for these effects so that it might be effectively targeted in the future to inhibit metastatic disease.

\section{Funding}

This work was supported by grants BiovitasPhD506726 (DT), Biovitas506546 (WY) from Biovitas Capital LTD and SerCymruPhD506724 (DT) from the Welsh Government.

\section{Acknowledgments}

We thank Mark Bishop, Jolene Twomey, Derek Scarborough and Luke Piggott for technical assistance.

\section{Conflict of Interest}

DT and WY salary and consumables was partially funded by Biovitas Capital LTD. 


\section{References}

1. Cumming, G., F. Fidler, and D.L. Vaux, Error bars in experimental biology. The Journal of Cell Biology, 2007. 177(1): p. 7.

2. Ridley, A.J., et al., Cell Migration: Integrating Signals from Front to Back. Science, 2003. 302(5651): p. 1704.

3. Roussos, E.T., J.S. Condeelis, and A. Patsialou, Chemotaxis in cancer. Nature Reviews Cancer, 2011. 11: p. 573.

4. $\quad$ Friedl, P., et al., Classifying collective cancer cell invasion. Nat Cell Biol, 2012. 14(8): p. 77783.

5. Parri, M. and P. Chiarugi, Rac and Rho GTPases in cancer cell motility control. Cell Communication and Signaling, 2010. 8(1): p. 23.

6. Zegers, M.M. and P. Friedl, Rho GTPases in collective cell migration. Small GTPases, 2014. 5: p. e28997.

7. Huang, B., et al., The three-way switch operation of Rac1/RhoA GTPase-based circuit controlling amoeboid-hybrid-mesenchymal transition. Sci Rep, 2014. 4: p. 6449.

8. Wilkinson, S., H.F. Paterson, and C.J. Marshall, Cdc42-MRCK and Rho-ROCK signalling cooperate in myosin phosphorylation and cell invasion. Nature Cell Biology, 2005. 7: p. 255.

9. Zhao, Z. and E. Manser, Myotonic dystrophy kinase-related Cdc42-binding kinases (MRCK), the ROCK-like effectors of Cdc42 and Rac1. Small GTPases, 2015. 6(2): p. 81-88.

10. Clark, A.G. and D.M. Vignjevic, Modes of cancer cell invasion and the role of the microenvironment. Current Opinion in Cell Biology, 2015. 36(Supplement C): p. 13-22.

11. Condeelis, J. and J.E. Segall, Intravital imaging of cell movement in tumours. Nature Reviews Cancer, 2003. 3: p. 921.

12. Farina, K.L., et al., Cell Motility of Tumor Cells Visualized in Living Intact Primary Tumors Using Green Fluorescent Protein. Cancer Research, 1998. 58(12): p. 2528-2532.

13. Tester, A.M., et al., MMP-9 secretion and MMP-2 activation distinguish invasive and metastatic sublines of a mouse mammary carcinoma system showing epithelialmesenchymal transition traits. Clinical \& Experimental Metastasis, 2000. 18(7): p. 553-560.

14. UK, C.R. Breast cancer statistics. 2012; Available from:

http://www.cancerresearchuk.org/health-professional/cancer-statistics/statistics-by-cancertype/breast-cancer\#heading-Zero

15. Gandalovicova, A., et al., Migrastatics-Anti-metastatic and Anti-invasion Drugs: Promises and Challenges. Trends Cancer, 2017. 3(6): p. 391-406.

16. Sleeman, J. and P.S. Steeg, Cancer metastasis as a therapeutic target. European Journal of Cancer, 2010. 46(7): p. 1177-1180.

17. Friedl, P. and K. Wolf, Tumour-cell invasion and migration: diversity and escape mechanisms. Nat Rev Cancer, 2003. 3(5): p. 362-374.

18. Sadok, A., et al., Rho kinase inhibitors block melanoma cell migration and inhibit metastasis. Cancer Res, 2015. 75(11): p. 2272-84.

19. Takeuchi, H., et al., Jasplakinolide: interaction with radiation and hyperthermia in human prostate carcinoma and Lewis lung carcinoma. Cancer Chemother Pharmacol, 1998. 42(6): p. 491-6.

20. Zhang, T., et al., Cucurbitacin E inhibits breast tumor metastasis by suppressing cell migration and invasion. Breast Cancer Res Treat, 2012. 135(2): p. 445-58.

21. Hart, I.R., A. Raz, and I.J. Fidler, Effect of cytoskeleton-disrupting agents on the metastatic behavior of melanoma cells. J Natl Cancer Inst, 1980. 64(4): p. 891-900.

22. Chen, X., et al., Bcl-3 regulates TGF[beta] signaling by stabilizing Smad3 during breast cancer pulmonary metastasis. Cell Death Dis, 2016. 7: p. e2508.

23. Wakefield, A., et al., Bcl3 selectively promotes metastasis of ERBB2-driven mammary tumors. Cancer Res, 2013. 73(2): p. 745-55. 
24. Kashatus, D., P. Cogswell, and A.S. Baldwin, Expression of the Bcl-3 proto-oncogene suppresses p53 activation. Genes Dev, 2006. 20(2): p. 225-35.

25. Westerheide, S.D., et al., The Putative Oncoprotein Bcl-3 Induces Cyclin D1 To Stimulate G(1) Transition. Molecular and Cellular Biology, 2001. 21(24): p. 8428-8436.

26. Thornburg, N.J., R. Pathmanathan, and N. Raab-Traub, Activation of nuclear factor-kappaB p50 homodimer/Bcl-3 complexes in nasopharyngeal carcinoma. Cancer Res, 2003. 63(23): p. 8293-301.

27. Legge, D.N., et al., BCL-3 promotes a cancer stem cell phenotype by enhancing 8-catenin signalling in colorectal tumour cells. Disease Models \&amp;amp; Mechanisms, 2019: $p$. dmm.037697.

28. $\mathrm{Wu}, \mathrm{L}$., et al., BCL3 expression promotes resistance to alkylating chemotherapy in gliomas. Sci Transl Med, 2018. 10(448).

29. Ahlqvist, K., et al., Expression of Id proteins is regulated by the Bcl-3 proto-oncogene in prostate cancer. Oncogene, 2013. 32(12): p. 1601-8.

30. Schwarz, E.M., et al., Immunological defects in mice with a targeted disruption in Bcl-3. Genes Dev, 1997. 11(2): p. 187-97.

31. Krakhmal, N.V., et al., Cancer Invasion: Patterns and Mechanisms. Acta Naturae, 2015. 7(2): p. 17-28.

32. Gao, J., et al., Integrative analysis of complex cancer genomics and clinical profiles using the cBioPortal. Sci Signal, 2013. 6(269): p. pl1.

33. Barretina, J., et al., The Cancer Cell Line Encyclopedia enables predictive modelling of anticancer drug sensitivity. Nature, 2012. 483(7391): p. 603-607.

34. Tan, T.Z., et al., Epithelial-mesenchymal transition spectrum quantification and its efficacy in deciphering survival and drug responses of cancer patients. EMBO Mol Med, 2014. 6(10): p. 1279-93.

35. Piccinini, F., A. Kiss, and P. Horvath, CellTracker (not only) for dummies. Bioinformatics, 2016. 32(6): p. 955-957.

36. Team, R.C., R: A Language and Environment for Statistical Computing. 2018.

37. Sun, W., C.T. Lim, and N.A. Kurniawan, Mechanistic adaptability of cancer cells strongly affects anti-migratory drug efficacy. J R Soc Interface, 2014. 11(99).

38. Stalker, L., J. Pemberton, and R.A. Moorehead, Inhibition of proliferation and migration of luminal and claudin-low breast cancer cells by PDGFR inhibitors. Cancer Cell International, 2014. 14: p. 89.

39. Zhao, J., et al., Mitochondrial dynamics regulates migration and invasion of breast cancer cells. Oncogene, 2013. 32(40): p. 4814-4824.

40. Kenny, P.A., et al., The morphologies of breast cancer cell lines in three-dimensional assays correlate with their profiles of gene expression. Molecular Oncology, 2007. 1(1): p. 84-96.

41. Gordon, L.A., et al., Breast cell invasive potential relates to the myoepithelial phenotype. Int J Cancer, 2003. 106(1): p. 8-16.

42. Lymperatou, D., et al., The exposure of breast cancer cells to fulvestrant and tamoxifen modulates cell migration differently. Biomed Res Int, 2013. 2013: p. 147514.

43. Upmanyu, N., et al., Impairment of the Gnalpha11-controlled expression of claudin-1 and MMP-9 and collective migration of human breast cancer MCF-7 cells by DHEAS. J Steroid Biochem Mol Biol, 2018. 182: p. 50-61.

44. Planas-Silva, M.D. and P.K. Waltz, Estrogen promotes reversible epithelial-to-mesenchymallike transition and collective motility in MCF-7 breast cancer cells. J Steroid Biochem Mol Biol, 2007. 104(1-2): p. 11-21.

45. Varankar, S.S. and S.A. Bapat, Migratory Metrics of Wound Healing: A Quantification Approach for in vitro Scratch Assays. Front Oncol, 2018. 8. 
46. Zhou, B., et al., Claudin 1 promotes migration and increases sensitivity to tamoxifen and anticancer drugs in luminal-like human breast cancer cells MCF7. Cancer Invest, 2015. 33(9): p. 429-39.

47. Scheel, C., et al., Paracrine and autocrine signals induce and maintain mesenchymal and stem cell states in the breast. Cell, 2011. 145(6): p. 926-40.

48. Liu, Y.L., et al., Assessing metastatic potential of breast cancer cells based on EGFR dynamics, in Sci Rep. 2019.

49. Biswas, D.K., et al., Epidermal growth factor-induced nuclear factor $\mathrm{KB}$ activation: A major pathway of cell-cycle progression in estrogen-receptor negative breast cancer cells, in Proc Natl Acad Sci U S A. 2000. p. 8542-7.

50. Nakshatri, H., et al., Constitutive activation of NF-kappaB during progression of breast cancer to hormone-independent growth. Mol Cell Biol, 1997. 17(7): p. 3629-39.

51. Pratt, M.A., et al., Estrogen withdrawal-induced NF-kappaB activity and bcl-3 expression in breast cancer cells: roles in growth and hormone independence. Mol Cell Biol, 2003. 23(19): p. 6887-900.

52. Keutgens, A., et al., The repressing function of the oncoprotein $B C L-3$ requires $C t B P$, while its polyubiquitination and degradation involve the E3 ligase TBLR1. Mol Cell Biol, 2010. 30(16): p. 4006-21.

53. Viatour, P., et al., GSK3-mediated BCL-3 phosphorylation modulates its degradation and its oncogenicity. Mol Cell, 2004. 16(1): p. 35-45.

54. Urban, B.C., et al., BCL-3 expression promotes colorectal tumorigenesis through activation of AKT signalling. Gut, 2016. 65(7): p. 1151-64.

55. Steeg, P.S., Targeting metastasis. Nature Reviews Cancer, 2016. 16: p. 201.

56. Anderson, R.L., et al., A framework for the development of effective anti-metastatic agents. Nature Reviews Clinical Oncology, 2019. 16(3): p. 185-204.

57. Cavallaro, U. and G. Christofori, Cell adhesion and signalling by cadherins and Ig-CAMs in cancer. Nat Rev Cancer, 2004. 4(2): p. 118-132.

58. Ridley, A.J., Rho GTPase signalling in cell migration. Current Opinion in Cell Biology, 2015. 36: p. 103-112.

59. Stengel, K. and Y. Zheng, Cdc42 in oncogenic transformation, invasion, and tumorigenesis. Cell Signal, 2011. 23(9): p. 1415-23.

60. Yamao, M., et al., Distinct predictive performance of Rac1 and Cdc42 in cell migration. Sci Rep, 2015. 5.

61. Wu, P.-H., D.M. Gilkes, and D. Wirtz, The Biophysics of 3D Cell Migration. https://doi.org/10.1146/annurev-biophys-070816-033854, 2016.

62. Chaudhary, S.C., et al., Shh and p50/Bc/3 signaling crosstalk drives pathogenesis of BCCs in Gorlin syndrome. Oncotarget, 2015. 6(34): p. 36789-36814.

63. Yamazaki, K., et al., Upregulated SMAD3 promotes epithelial-mesenchymal transition and predicts poor prognosis in pancreatic ductal adenocarcinoma. Lab Invest, 2014. 94(6): p. 68391.

64. Tan, E.J., et al., Regulation of transcription factor Twist expression by the DNA architectural protein high mobility group A2 during epithelial-to-mesenchymal transition. J Biol Chem, 2012. 287(10): p. 7134-45. 
Fig. 1 Breast cancer cell lines exhibit distinct modes of migration. (A) Representative images of MDA-MB-436, MDAMB-231 and MCF-7 cell morphology and Bcl-3 localization using immunofluorescence for the actin cytoskeleton marker phalloidin (Red) and Bcl-3 (Green) (scale bar $50 \mu \mathrm{m}$ ). Migration assays were used to determine the migration capacity of each cell line (B) Transwell assays compared chemotaxis migration, (C) time-lapse microscopy compared random single cell migration and (D) wound healing assays compared collective migration. Error bars represent $\pm S E M$ of $n=3$ (RMANOVA, $* * *=p<0.001, * * * *=p<0.0001)$. The EMT scores of 56 breast cancer cell lines were calculated with each line grouped into epithelial, intermediate or mesenchymal subtypes. (E) Comparison of CDH1 expression in each group confirmed loss of expression in mesenchymal-like cells; with (F) no difference in Bcl-3 expression observed between each group. Error bars represent \pm SEM epithelial $n=26$, intermediate $n=15$, mesenchymal $n=15 \quad(t$ test, $* * *=p<0.001$, $* * * *=p<0.0001$ ). (G) Bcl-3 expression in MDA-MB-436, MDA-MB-231 and MCF-7 cells was determined by qRT-PCR with each cell lines relative random single cell migration plotted alongside to highlight the correlation of Bcl-3 expression and this form of motility. Error bars for qRT-PCR data represent confidence intervals $n=3$ with interval overlap used to determine significance as described in $[1],(* *=\mathrm{p}<0.01)$.

Fig. 2 Suppression of Bcl-3 inhibits three forms of cell migration. (A) MDA-MB-436 cells were treated with Bcl-3 siRNA before seeding into Transwell assays to assess chemotaxis driven single cell migration resulting in reduced motility compared to scRNA treated controls. (B\&C) This effect was also observed in MDA-MB-231 and MCF-7 cells. (D\&E) MDA-MB-436 and MDA-MB-231 cells were also analysed by time-lapse and CellTracker software for changes in random single cell migration with Bcl-3 siRNA treatment resulting in reduced migration in both cell lines. Collective migration was reduced following Bcl-3 siRNA treatment in (F) MDA-MB-436, (G) MDA-MB-231 and (H) MCF-7 cells. Error bars represent \pm SEM of $n=3($ RM-ANOVA, $*=p<0.05, * *=p<0.01 * * *=p<0.001)$.

Fig. 3 Bcl-3 suppression potentiates EMT-mediated migration. MCF-7 cells were treated with EMT inducing supplement (M-EMT). (A) Representative images of MCF-7, M-EMT treated MCF-7 (EMT MCF-7) and EMT MCF-7 cells following the removal of M-EMT for 6 days (MET MCF-7). Immunofluorescence for the actin cytoskeleton marker phalloidin (Red), the DNA marker DAPI (Blue) and the cell adhesion molecule E-cadherin (Green) highlighting the loss of cell-cell contacts to a more mesenchymal-like morphology following EMT stimulation (scale bar $50 \mu \mathrm{m}$ ). (B) Bcl-3 expression was upregulated following M-EMT treatment as determined by qRT-PCR. Error bars represent confidence intervals of 3 independent experiments with significance determined by confidence overlap as described in [1], $(* *=\mathrm{p}<0.01)$. (C) The number of motile single cells migrating away from the collective front following wound-healing assays was determined using time-lapse microscopy, with M-EMT treatment increasing the number of single migratory cells. (D) Representative images of MCF-7 and EMT MCF-7 cells 24h after wounds were created. (E) The number of single cells migrating away from the collective front in EMT MCF-7 cells was quantified following Bcl-3 suppression, which resulted in a reduced number of motile single cells. (F) Chemotaxis driven migration was inhibited in EMT MCF-7 cells treated with Bcl-3 siRNA. (G) Collective migration was also reduced following Bcl-3 suppression in wound healing assays. Error bars represent \pm SEM of $\mathrm{n}=3$ experiments. (RM-ANOVA, $* *=\mathrm{p}<0.01, * * *=\mathrm{p}<0.001$ ).

Fig. 4 Suppression of Bcl-3 inhibits migration through loss of Cdc42 activity. (A) Cdc42 expression was reduced in breast cancer cell lines following treatment with Bcl-3 siRNA, determined by qRT-PCR $\mathrm{n}=3(*=\mathrm{p}<0.05, * *=\mathrm{p}<0.01)$. (B)

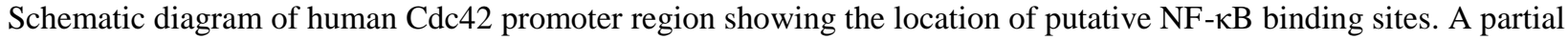
sequence of the Cdc42 promoter is shown highlighting NF- $\kappa$ B 1 binding sites (Green and Blue) and the region amplified by PCR (line above binding site sequences) following p50 IP. Pol 2 indicates binding region of RNA polymerase 2 (ENCODE) and represents the transcription initiation region. (C) ChIP assays were performed using anti-p50 antibody or IgG control following Bcl-3 siRNA treatment in 231L cells. qRT-PCR was used to detect NF-kB specific binding to the Cdc42 promoter with Bcl-3 siRNA resulting in a loss of p50 binding. Data shown is representative of 3 independent experiments. (D) NF- $\mathrm{BB}$ reporter assays following Bcl-3 siRNA showed a reduction in NF- $\kappa \mathrm{B}$ activity $\mathrm{n}=4(t$ test, $*=\mathrm{p}<0.05$ ). (E) G-LISA GTPase activation assays determined a reduction in active Cdc42 following Bcl-3 suppression $\mathrm{n}=$ 3 (RM ANOVA, $* * *=p<0.001$ ). (F) Increased CFL2 expression was determined by qRT-PCR in each cell line $\mathrm{n}=3$ $(*=\mathrm{p}<0.05, * *=\mathrm{p}<0.01)$. (G) Chemotaxis driven migration was reduced in $231 \mathrm{~L}$ cells following treatment with Cdc 42 siRNA. Cdc42 knockdown also reduced (H) random single cell and (I) collective migration in MDA-MB-436 and MCF-7 cells respectively with no additional effect on either form of migration following combined inhibition with Bcl-3 siRNA (RM-ANOVA, $* *=p<0.05, * * *=p<0.001)$. qRT-PCR error bars represent confidence intervals of 3 independent experiments with significance determined by confidence overlap as described in [1]. 
Fig. 5 Bcl-3 overexpression increases single cell migration and metastasis. (A) WT Bcl-3 and ANK mutant Bcl-3 with disrupted NF- $\kappa$ B binding were overexpressed in 231L and MDA-MB-436 cells, represented by western blot against Bcl-3 (B) NF- $\kappa$ B reporter assays showed increased NF- $\kappa$ B activity in WT overexpressing cells while activity was reduced in ANK overexpressing cells, $\mathrm{n}=4$ ( $t$ test, $*=\mathrm{p}<0.05$, $* * \mathrm{p}<0.01)$. Migration assays were performed in each cell line with WT Bcl-3 overexpression resulting in increased single cell motility in both (C) chemotaxis driven and (D) random migration assays whilst also reducing (E) collective migration, while ANK overexpression inhibited all 3 forms of motility, $n=3$ (RM-ANOVA, $*=\mathrm{p}<0.05, * *=\mathrm{p}<0.01 * * *=\mathrm{p}<0.001)$. (F) The number of metastatic legions per mouse was represented for mice injected with $2 \times 10^{5}$ MDA-MB-231-Luc cells with overexpression of WT Bcl-3 increasing metastatic burden compared to controls while ANK overexpression reduced metastasis incidence rate, $\mathrm{n}=5(t$ test, $*=\mathrm{p}<0.05)$. (G) Representative images of H\&E stained metastatic legions from mice injected with either WT/ANK overexpressing Bcl-3 or 231L cells pre-treated with Bcl-3 siRNA. (H) Metastatic incidence rate in the lungs and livers of mice injected with $231 \mathrm{~L}$ cells following pre-treatment of Bcl-3 siRNA which reduced metastatic burden compared to scRNA controls, $\mathrm{n}=4(t$ test, $*=\mathrm{p}<0.05)$. 